Research, Society and Development, v. 9, n. 5, e72953102, 2020

(CC BY 4.0) | ISSN 2525-3409 | DOI: http://dx.doi.org/10.33448/rsd-v9i5.3102

\title{
Prevalência de alterações prostáticas em idosos
}

Prevalence of prostatic changes in the elderly

Prevalencia de cambios prostáticos en ancianos

Recebido: 19/03/2020 | Revisado: 20/03/2020 | Aceito: 27/03/2020 | Publicado: 28/03/2020

Cenir Gonçalves Tier

ORCID: https://orcid.org/0000-0003-1539-7816

Universidade Federal do Pampa, Brasil

E-mail: cgtier@gmail.com

Roberta Hilgert Recart

ORCID: https://orcid.org/0000-0002-4321-2738

Hospital Santa Casa de Uruguaiana, Brasil

E-mail: hilgert_roberta@gmail.com

Letice Dalla Lana

ORCID: https://orcid.org/0000-0002-9624-8152

Universidade Federal do Pampa, Brasil

E-mail: leticedl@hotmail.com

Bruna Sodré Simon

ORCID: https://orcid.org/0000-0003-3855-1310

Universidade Federal do Pampa, Brasil

E-mail: enf.brusimon@gmail.com

Raquel Pötter Garcia

ORCID: https://orcid.org/0000-0002-5503-7981

Universidade Federal do Pampa, Brasil

E-mail: raquelgarcia@unipampa.edu.br

Bruna Gomes Furtado

ORCID: https://orcid.org/0000-0002-4327-1973

Secretaria Municipal de Saúde de Uruguaiana, Brasil

E-mail: brunacfurtado@gmail.com

\section{Resumo}

Objetivo: Identificar a prevalência dos tipos de alteração prostática em idosos, diagnosticadas por meio da biópsia. Metodologia: Pesquisa transversal e documental realizada entre julho e outubro de 2016 em 230 prontuários de idosos que realizaram biópsia prostática em um 
laboratório de patologia público na Região Oeste do Rio Grande do Sul. Foram analisadas as variáveis ano e tipo de alteração. Os dados foram tabulados em planilha do Excel e submetidos à análise estatística descritiva. Resultados: A prevalência de hiperplasia benigna prostática foi de 123 (53\%) e de adenocarcinoma de 107 (46\%). Conclusão do estudo: A prevalência de adenocarcionama aumentou no ano de 2015, porém no período analisado o maior número de casos são de idosos com Hiperplasia Benigna Prostática. Nota-se a importância da realização do exame diagnóstico, dados os elevados índices de alterações prostáticas, e a necessidade de desenvolver ações voltadas para o fortalecimento da Política Nacional de Atenção Integral à Saúde do Homem.

Palavras-chave: Idoso; Doenças prostáticas; Saúde do homen; Enfermagem.

\section{Abstract}

Objective: To identify the prevalence of prostate alteration types in the elderly, diagnosed by biopsy. Method and Materials: Cross-sectional and documentary research conducted between July and October 2016 on 230 medical records of the elderly who performed prostate biopsy in a public Pathology Laboratory in the West Region of Rio Grande do Sul. Year and type of change were analyzed as variables. Data were tabulated in Excel spreadsheet and submitted to statistical analysis. Results: The prevalence of benign prostatic hyperplasia was $123(53 \%)$ and adenocarcinoma was 107 (46\%). Conclusion: The prevalence of adenocarcionama increased in 2015, but in the period analyzed the largest number of cases are elderly people with Benign Prostatic Hyperplasia. It is noted the importance of performing of the diagnostic examination, given the high numbers of prostate alterations, and the need to development actions aimed at strengthening the National Policy for Integral Attention to Men's Health.

Keywords: Elderly; Prostatic diseases; Men's health; Nursing.

\section{Resumen}

Objetivo: identificar la prevalencia de los tipos de anomalías prostáticas en los ancianos diagnosticados mediante biopsia. Metodología: Investigación transversal y documental realizada entre julio y octubre de 2016 en 230 registros de personas mayores que se sometieron a biopsia de próstata en un laboratorio público de patología en la Región Oeste de Rio Grande do Sul. Se analizaron las variables año y tipo de estudio. cambio Los datos se tabularon en una hoja de cálculo de Excel y se sometieron a un análisis estadístico descriptivo. Resultados: La prevalencia de hiperplasia prostática benigna fue 123 (53\%) y el 
adenocarcinoma fue 107 (46\%). Conclusión del estudio: la prevalencia de adenocarcionama aumentó en 2015, pero en el período analizado el mayor número de casos son personas de edad avanzada con hiperplasia prostática benigna. Se observa la importancia de llevar a cabo el examen de diagnóstico, dada la gran cantidad de cambios prostáticos, y la necesidad de desarrollar medidas encaminadas a fortalecer la Política Nacional para la Atención Integral a la Salud de los Hombres.

Palabras clave: Ancianos; Enfermedades prostáticas; Salud masculina; Enfermería.

\section{Introdução}

O Estatuto do Idoso, Lei $n^{\circ} 10.741$, de $1^{\circ}$ de outubro de 2003, art. 15, assegura uma atenção integral à saúde do idoso, articulada e contínua das ações e serviços, para a prevenção, a promoção, a proteção e a recuperação da saúde por intermédio do Sistema Único de Saúde (Brasil, 2003). Neste ínterim, a pessoa idosa tem acesso à prevenção e tratamento de alterações potencializadas no processo de envelhecimento.

Dentre as alterações provocadas pelo processo de envelhecer, destaca-se a modificação nas células do corpo humano, que conduzem a maior suscetibilidade ao câncer (Brasil, 2019). A idade é o maior fator de risco no câncer de próstata, uma vez que a incidência quanto à mortalidade aumenta significativamente após os 50 anos. $\mathrm{O}$ câncer de próstata é o segundo mais comum entre os homens, atrás apenas do câncer de pele não melanoma, sendo que cerca de $75 \%$ dos casos no mundo ocorrem a partir dos 65 anos (Brasil, 2019).

A detecção do câncer de próstata, na maioria das vezes, ocorre após identificação de sinais e sintomas pelo idoso, os quais já estão em fase avançada da doença (Santos \& Lamounier, 2013). Tal fato pressupõe que as ações como o Novembro Azul, voltadas para a saúde do homem ainda estão incipientes.

Por outro lado, o avanço dos métodos diagnósticos, pelo progresso dos sistemas de informação e pelo aumento da expectativa de vida do brasileiro auxiliam na detecção das patologias como hiperplasia benigna da próstata (HBP), que acomete em torno de $10 \%$ dos homens a partir dos 30 anos de idade e $90 \%$ dos idosos com 90 anos; a HBP pode causar problemas urinários e comprometer a qualidade de vida desses indivíduos (Crippa \& Dall'oglio, 2013). O percentual de idosos que realizam tratamento quimioterápico para o câncer de próstata atinge 17\% com predominância de indivíduos entre 60 e 80 anos de idade, 
com baixa escolaridade, casados ou em união estável, e com baixa renda familiar (Santos, et al., 2017).

Mesmo identificando as ações que facilitam e ampliam o acesso da população masculina aos serviços de saúde no Brasil, a incidência por câncer de próstata nos indivíduos com idade entre 40 e 60 anos foi de 0,42 casos para cada 1000.00 homens avaliados em 2013 (Brasil, 2009; 2013). Acreditando que este valor pode ser subestimado, tendo em vista as neoplasias benignas e óbitos sem causa definida, este estudo busca identificar a prevalência dos tipos de alteração prostática em idosos que realizaram a biópsia.

\section{Metodologia}

Trata-se de uma pesquisa de campo, quantitativa, transversal e documental (Pereira, et al., 2018), realizada em um laboratório de patologia público da Região Oeste do Rio Grande do Sul. A coleta de dados ocorreu nos 230 prontuários pertencentes a homens idosos que realizaram biópsia prostática de janeiro de 2012 a dezembro 2015. A limitação temporal refere-se à delimitação do sistema de registros das biópsias.

Como critérios de inclusão, adotou-se os prontuários de idosos (idade igual ou maior que 60 anos) que realizaram biópsia prostática e obtiveram algum diagnóstico médico. Excluíram-se os prontuários incompletos. Foram analisadas as variáveis ano e tipo de alteração.

Após coletados, os dados foram tabulados em uma planilha no Excel e então submetidos à análise estatística descritiva. Esta pesquisa respeitou os preceitos éticos relacionados à pesquisa com seres humanos conforme Resolução nº66/2012 do Conselho Nacional de Saúde e recebeu aprovação do Comitê de Ética em Pesquisa (CAAE 48592115.9.0000.5323).

\section{Resultados}

Foram avaliados 230 prontuários de homens idosos entre 60 e 90 anos de idade referente ao período de 2012 a 2015. Conforme a Tabela 1, constatou-se que a prevalência de hiperplasia benigna prostática foi de 123 (53,5\%) e de adenocarcinoma 107 (46,5\%).. O ano de 2015 teve maior prevalência de hiperplasia benigna prostática 35 (46\%) e adecarcionama $41(54 \%)$. 
(CC BY 4.0) | ISSN 2525-3409 | DOI: http://dx.doi.org/10.33448/rsd-v9i5.3102

Tabela 1. Prevalência de hiperplasia benigna prostática e de adenocarcinoma, Uruguaiana, 2016.

\begin{tabular}{cccc}
\hline Ano & $\mathbf{n}^{\mathbf{0}}$ idosos & $\begin{array}{c}\text { Prevalência Hiperplasia } \\
\text { Benigna Prostática }\end{array}$ & $\begin{array}{c}\text { Prevalência } \\
\text { Adenocarcinoma }\end{array}$ \\
\hline $\mathbf{2 0 1 2}$ & 76 & $40(52,6 \%)$ & $36(47,4 \%)$ \\
$\mathbf{2 0 1 3}$ & 33 & $23(69,6 \%)$ & $10(30,3 \%)$ \\
$\mathbf{2 0 1 4}$ & 45 & $25(55,5 \%)$ & $20(44,4 \%)$ \\
$\mathbf{2 0 1 5}$ & 76 & $35(46 \%)$ & $41(54 \%)$ \\
Total & 230 & $123(53,5 \%)$ & $107(46,5 \%)$ \\
\hline
\end{tabular}

Fonte: Dados da Pesquisa, 2019.

\section{Discussão}

O estudo revelou que a prevalência de hiperplasia benigna prostática foi de 123 (53,5\%) e de adenocarcionoma 107 (46,5\%) para adenocarcinoma. A taxa de incidência é maior nos países desenvolvidos em comparação aos países em desenvolvimento, a estimativa é de 68.220 novos casos por ano no Brasil. Sendo que ano de 2017 no Brasil foram registrados 15.391 mortes por câncer de próstata (Brasil, 2019). Todavia o aumento observado nas taxas de incidência no Brasil pode ser parcialmente justificado pela evolução dos métodos diagnósticos (exames), pela melhoria na qualidade dos sistemas de informação e pelo aumento da expectativa de vida.

Este estudo revelou que em 2015 o diagnóstico de adenocarcinoma foi mais elevado 41 (54\%) em comparação com os demais anos pesquisados, superando a Hiperplasia Benigna Prostática $35(46 \%)$. Contudo estes achados podem manter relação com a maior procura pelo serviço de diagnóstico.

Mais precisamente, pode-se pressupor que ainda existem dificuldades políticas organizacionais para a prevenção do câncer de prostáta, que podem estar associadas a dificuldades socioculturais da polução, e ao acesso do usuário ao diagnóstico e tratamento.

Agregado, infere-se que a alimentação rica em gordura, característica da região estudada, excesso de gordura corporal também podem ter sido um dos fatores de risco que propiciou o maior percentual de câncer prostático em homens idosos nesta região em relação a outros estudos.

Assim como revelado nesta pesquisa, a hiperplasia benigna prostática é a condição patológica mais comum nos homens idosos e a segunda causa de intervenção cirúrgica em 
homens com mais de 60 anos de idade (Silva, 2011). Entretanto, vale destacar, que a hiperplasia benigna prostática vem sendo amplamente diagnosticada, pois seu principal sintoma é a dificuldade relatada pelos homens ao urinar (Farias Filho, et al., 2017). Porém, pode-se afirmar que o diagnóstico da doença é tardio, pois as manifestações ocorrem quando há obstrução parcial ou total da uretra, causando a dificuldade de urinar, jato urinário fraco e sensação de não esvaziamento da bexiga (Santos \& Lamounier, 2013).

Mesmo reconhecendo que o Brasil é o primeiro país da América Latina e o segundo do continente americano a implementar uma Política Nacional De Atenção Integral à Saúde do Homem (Brasil, 2009) que dispõe sobre a instituição do Programa Nacional de Controle do Câncer de Próstata (Brasil, 2001), observa-se que a prevenção ainda é incipiente. Mais precisamente, os homens idosos não apresentavam acompanhamento preventivo haja vista a alta prevalência de câncer diagnosticado pelo procedimento de biópsia. Essa constatação sustenta-se quando o tipo de serviço de saúde preventivo utilizado pelos homens idosos para os exames de testagem do Antígeno Prostático Específico (PSA) e toque retal são ainda pouco estudados e realizados pela população brasileira (Bibiano, et al., 2019).

O baixo percentual de homens sintomáticos, revela a importância de realizar exames diagnósticos mesmo sem a presença de sintomas e sinais. A detecção precoce poderá prevenir ou retardar o tratamento para a hiperplasia prostática benigna com fármacos e procedimentos cirúrgicos (Farias Filho, et al., 2017).

No que tange à prevenção do câncer de próstata, a Sociedade Brasileira de Urologia recomenda que a partir dos 50 anos a população masculina realize os exames de prevenção. Porém, aqueles que são da raça negra ou com parentesco do primeiro grau que teve ou tem o câncer deve começar a prevenção a partir dos 40 anos (Brasil, 2019).

A identificação de homens com idade entre 60 a 90 anos inferem que a alteração prostática pode ocorrer tardiamente, pois pesquisas apontam que a hiperplasia pode atingir nas idades superiores a 45 anos até 79 anos de idade (Dantas, et al., 2018), bem como de 43 a 93 anos de idade (Castro, et al., 2011).

Mesmo que este estudo tenha utilizado apenas acima de 60 anos, os resultados inferem à resistência dos homens na realização dos exames de prevenção, o que leva uma procura tardia quando os sintomas já estão instaurados. Dessa forma, acredita-se que culturalmente os homens procuram pouco os serviços, devido o preconceito, medo, sustentando pensamentos e ações previamente formados e que os impedem de buscar a prevenção do câncer de próstata e, se necessário, até mesmo o tratamento eficiente (Costa \& Moura, 2013, Czorny, et al., 2017). 
Research, Society and Development, v. 9, n. 5, e72953102, 2020

(CC BY 4.0) | ISSN 2525-3409 | DOI: http://dx.doi.org/10.33448/rsd-v9i5.3102

Há uma relutância dos homens quanto ao exame preventivo da próstata e, com isso, os profissionais da atenção primária devem orientá-los e aumentar a adesão aos serviços de saúde. Isso facilita a detecção e tratamento precoce desse tipo de câncer, reduzindo a mortalidade pela doença (Czorny, et al., 2017). Na Atenção primária à saúde, diversos espaços podem servir de cenário para promoção da saúde, como o ambiente escolar, centros comunitários, igrejas e pastorais (Santos Ribeiro, et al., 2019).

No entanto, vale salientar a importância do acompanhamento multiprofissional voltado não apenas para a detecção adequada deste usuário, mas principalmente para promover a gestão do tratamento de forma contínua até a alta do quadro clínico (Makki, et al., 2018). Além disso, tal monitoramento facilita o encaminhamento destes homens aos serviços de média e alta complexidade, sendo a atenção básica a porta de entrada e referência organizacional para a demanda dos serviços (Krüger \& Cavalcanti, 2018).

Nesse contexto reforça-se a importância da gestão municipal, estadual e federal viabilizar o acesso ao diagnósticos e tratamento das alterações prostáticas. Ademais se faz fundamental que os profissionais de saúde potencializarem a oferta de orientações referentes à saúde do homem, bem como identificar fatores de riscos e sintomas associados as alterações prostáticas.

Como limitações do estudo destacam-se o sistema de informações ainda incipiente, a baixa qualidade de registros nos prontuários dos pacientes, o curto período de tempo estudado, e a inconstância na oferta do serviço pelo SUS na região estudada.

\section{Conclusões}

A prevalência de hiperplasia beningna prostática foi de $123(53,5 \%)$ e de adenocarcinoma 107 (46,5\%) no período estudado.

Neste contexto, reforça-se a necessidade dos profissionais de saúde orientarem em relação ao diagnóstico precoce do câncer de próstata, bem como realizar ações voltadas para o fortalecimento da Política Nacional de Atenção Integral à Saúde do Homem.

Sugere-se que sejam realizados outros estudos a fim de evidenciar boas práticas que despertem a busca diagnóstica, mesmo na ausência de sinais e sintomas pelos idosos, bem como compreender o fluxo destes usuários na rede de cuidado. 


\section{Referências}

Bibiano, A. M. B., Moreira, R. S., Tenório, M. M. G. O. \& Silva, V.L. (2019). Fatores associados à utilização dos serviços de saúde por homens idosos: uma revisão sistemática da literatura. Ciênc. saúde coletiva, 24(6), 2263-2278.

Brasil. (2003). Estatuto do idoso: lei federal n 10.741, de 01 de outubro de 2003. Brasília: Ministério da Saúde. Disponivel em: http://www.planalto.gov.br/ccivil_03/leis/2003/110.741.htm. Acesso em 24 de março de 2020

Brasil. (2009). Política Nacional de Saúde do Homem. Brasília: Ministério da Saúde.

Brasil. (2019). Câncer de próstata. Ministério da Saúde. Instituto nacional de câncer (INCA).

Brasília: Ministério da Saúde. Disponivel em: https://www.inca.gov.br/tipos-decancer/cancer-de-prostata. Acesso em 24 de março de 2020

Brasil. Lei n. 10.289, de 20 de setembro de 2001. Dispõe sobre a Instituição do Programa Nacional de Controle do Câncer de Próstata. Legislação Federal. Brasília: Ministério da Saúde; 2001.

Brasil.(2013). Instituto brasileiro de estatística e geografia (IBGE). Brasília: Ministério da Saúde. Disponível em http://www.ibge.gov.br/home/. Acesso em 24 de março de 2020

Castro, H. A. S., Iared, W., Shigueoka, D. C., Mourão, J. E. \& Ajzen, S. (2011). Contribuição da densidade do PSA para predizer o câncer da próstata em pacientes com valores de PSA entre 2,6 e 10,0 ng/ml. Radiol Bras, 44(4), 205-209.

Costa, T. B. \& Moura, V. F. (2013). O significado do toque retal da próstata para o homem: enfermeiro na promoção da saúde. Rev. pesqui. cuid. Fundam, 5(4), 537- 546.

Crippa, A. \& Dall'oglio M. (2013) Como Diagnosticar e Tratar Doenças da Próstata. RBM, Dez., 70(12), 47-56. 
Czorny, R. C. N., Pinto, M. H., Pompeo, D. A., Bereta, D., Cardoso \& L. V., Silva, D.M. (2017). Fatores de risco para o câncer de próstata: população de uma unidade básica de saúde. Cogitare Enfermagem, 22(4).

Dantas, A. S., Moura, S. G., Alburquerque KF, Gerônimo VS, Andrade J. H. \& Dantas MS. (2018). Representações sociais de agentes comunitários de saúde acerca do câncer de próstata. Revista de Pesquisa: Cuidado é Fundamental Online, 10(1): 145-152.

Farias Filho, R. F., Rocha, A. S., Costa, A. M. C. B., Ricardo, F. I. S., Sousa, G. S. \& Santos, T. C. (2017). Hiperplasia prostática benigna: revisão de literatura. $R$. Interd, 10(1), 200-204.

Krüger, F. P. G.\& Cavalcanti, G. (2018). Câncer de Próstata no Brasil: Revisão Integrativa. Revista Brasileira de Cancerologia, 64(4), 561-567.

Makki, H., Reckziegel, J. C. L., Agostinetto, L. \& Pereira, R. C. (2018). Política Nacional de Atenção Integral à Saúde do Homem: elaborando estratégias de educação em saúde. Revista UNIPLAC, 6(1), e18.

Pereira, A.S. et al. (2018). Metodologia da pesquisa científica. [e-book]. Santa Maria. Ed. UAB/NTE/UFSM. Disponível em: https://repositorio.ufsm.br/bitstream/handle/1/15824/Lic_Computacao_MetodologiaPesquisa-Cientifica.pdf?sequence=1. Acesso em: 24 março 2020.

Santo Ribeiro, E. E., de Carvalho, M. R., Caminha, J. M., Gomes, I. S \& Silva Melo, S. S. (2019). Práticas de enfermagem na promoção da saúde do homem: revisão integrativa. Revista Eletrônica Acervo Saúde, (26), e460.

Santos, C. L. \& Lamounier, T. A. C. (2013) Aspectos clínicos e laboratoriais do câncer de próstata. Acta de Ciências e Saúde, 02(01), 32-49

Santos, E. G. A, Souza, J. C., Santos, A. L. S., Santos, M. I. P. O. \& Oliveira, T. N. C. (2017) Perfil clínico-epidemiológico de idosos submetidos à quimioterapia antineoplásica atendidos em um hospital de referência oncológica do estado do Pará, Brasil. Rev Pan-Amaz Saúde, $8(2), 45-54$. 
Silva, W. B. (2011). Explorando o conhecimento dos usuários de duas unidades de saúde da família sobre a hiperplasia benigna prostática. [Trabalho de conclusão de curso]. Campina Grande/Paraíba: Universidade Estadual da Paraíba.

\section{Porcentagem de contribuição de cada autor no manuscrito}

Cenir Gonçalves Tier - 18\%

Roberta Hilgert Recart - 18\%

Letice Dalla Lana - $16 \%$

Bruna Sodré Simon - 16\%

Raquel Pötter Garcia - 16\%

Bruna Gomes Furtado - 16\% 